\title{
GO NATURAL, SAY NO TO CHEMICALS - A SYSTEMATIC REVIEW ON EFFECTIVENESS OF GREEN TEA EXTRACT CONTAINING FORMULATIONS ON DENTAL CARIES
}

\author{
JAYASHRI PRABAKAR*, JOSEPH JOHN, MEIGNANA ARUMUGHAM I, PRADEEP KUMAR R, SRISAKTHI D
}

Department of Public Health Dentistry, Saveetha Dental College, Saveetha Institute of Medical and Technical Sciences, Saveetha University, Chennai, Tamil Nadu, India. Email: shrijaya2009@gmail.com

Received: 21 April 2018, Revised and Accepted: 28 September 2018

\section{ABSTRACT}

Objective: In recent years, the side effects encountered with the use of chemicals has led to the search for safe anticariogenic alternatives. Green tea is one such naturally occurring alternative which possesses anticariogenic activity through a direct bactericidal effect against cariogenic microorganisms. Therefore, the objective of this systematic review was to analyze the existing literature on the effectiveness of green tea extract containing formulations on dental caries.

Methods: The databases of PubMed, Cochrane, Science Direct, Metapress, and Google Scholar were searched up to July 2015 for the related topic. References of the selected articles and relevant reviews were searched for any missed publications.

Results: The systematic search revealed a total of 437 publications which were scrutinized based on eligibility criteria. Six publications fulfilled the criteria and 431 publications were excluded from the review. Out of six studies, three studies reported a statistically significant difference in favor of green tea while two studies showed no statistically significant difference between the groups. One study reported no statistically significant difference between the two groups; however, the reduction observed in chlorhexidine (control group) was slightly greater than green tea.

Conclusion: With the available evidence, it can be concluded that green tea extract containing formulations are effective in reducing cariogenic microflora and caries increment.

Keywords: Dental caries, Cariogenic microflora, Green tea.

(C) 2019 The Authors. Published by Innovare Academic Sciences Pvt Ltd. This is an open access article under the CC BY license (http://creativecommons. org/licenses/by/4. 0/) DOI: http://dx.doi.org/10.22159/ajpcr.2019.v12i2.26835

\section{INTRODUCTION}

Dental caries is the most important and widely spread dental diseases with its prevalence declining gradually in many developed countries, but it continues to be a major public health problem in industrialized countries and has become an emerging public health problem in developing countries [1]. In developing countries like India, caries prevalence has shown results ranging from $31.5 \%$ to $89 \%$ [2-6]. Keyes identified primary caries inducing factors as the teeth, the microflora, and the substrate [7]. Several approaches have been adopted to prevent dental caries, one of these approaches include the elimination of cariogenic bacteria [8].

Oral cavity harbors a wide variety of microorganisms; these are considered crucial for the initiation and progression of dental diseases [9]. Over 400 species of microbes inhabit as commensals in the oral cavity of a healthy adult [10]. An aberration to this ecology due to dietary habits, improper oral hygiene, or systemic factors leads to increased cariogenic microorganisms [11].

Dental caries is a multi-factorial disease in which, Streptococcus mutans and lactobacilli in dental plaque, play an important role [12]. Therefore, dental caries is defined as a multi-factorial, transmissible, and infectious oral disease caused primarily by the complex interaction of cariogenic oral flora (biofilm) with fermentable dietary carbohydrates on the tooth surface over time [13].

Cariogenic microorganisms such as S. mutans and Lactobacillus acidophilus are the primary causative microorganism for the development of dental caries. These cariogenic microorganisms encourage the accumulation and adherence of plaque biofilm by metabolizing sucrose into sticky glucan.

Dental plaque is a bacterial biofilm adhering to the tooth surfaces. It is mainly composed of complex bacterial populations organized in a carbohydrate matrix also containing a small number of epithelial cells, leukocytes, macrophages, and inorganic components such as calcium and phosphorus [14]. The microorganisms in dental plaque degrade the dietary carbohydrates producing lactic acid leading to localized demineralization and the eventual formation of dental caries $[15,16]$. Plaque-induced caries is a local disease; therefore, the local use of antimicrobial agents is more efficient than their systemic use [17].

The prevention of dental caries in children and adolescents is generally regarded as a priority for dental services and considered more costeffective than its treatment [18]. Fluoride therapy has been the centerpiece of caries-preventive strategies since the introduction of water fluoridation schemes over five decades ago [19]. Even though fluoride therapy was considered to be gold standard for prevention of dental caries; however, concern has been expressed that dental fluorosis, enamel defects caused by young children chronically ingesting excessive amounts of fluoride during the period of tooth formation (up to the age of 6 years), is increasing in both fluoridated and non-fluoridated communities, and the early use of fluoridated toothpaste by young children may be an important risk factor [20,21]. Chronic ingestion of fluoride from toothpaste in children is common $[22,23]$ and despite the large variation in the amount swallowed, the younger children are, the more likely they are to swallow larger amounts, which often represent a substantial part of the total daily fluoride intake and can be enough to cause fluorosis [24,25].

Hence, to overcome the adverse effects of fluoridated toothpaste, various naturally derived antimicrobial agents are incorporated to dentifrices and mouth rinses to curb cariogenic microorganisms and to prevent the formation of dental caries. There has been renewed interest in naturally occurring products in recent years [26], and also, the side effects encountered with the use of synthetic formulations have led to the surge for novel and safe alternatives [11]. 
Green tea is one such natural alternative which possesses anticariogenic activity through a direct bactericidal effect against cariogenic microorganisms and indirectly by prevention of bacterial adherence to teeth [27].

Several studies have indicated that bioactive components of green tea are able to influence the process of caries formation through several different mechanisms: They may inhibit proliferation of the streptococcal agent, interfere with the process of bacterial adhesion to tooth enamel, and act as inhibitors of glucosyltransferase and amylase [27-30].

The beneficial effects of green tea are generally attributed to its polyphenol content, particularly catechins which have got diverse pharmacological properties that include antibacterial effects, anticariogenic effects, and antioxidative effects [31].

The four major catechins are epigallocatechin-3-gallate which represents approximately $59 \%$ of the total catechins, epigallocatechin (19\% approximately), epicatechin-3-gallate (13.6\% approximately), and epicatechin (6.4\% approximately) [32]. Green tea being the nonfermented type possesses more Catechin than Oolong tea (semifermented) and Black tea (fermented) [33]. Rasheed and Haider [28] described the antibacterial effect of green tea catechins against $S$. mutans bacteria and stated that catechins were of great value in the reduction of $S$. mutans bacteria and caries prevalence. Maryam [34] and Ferrazzano [35] showed a significant reduction of colony counts of salivary S. mutans and Lactobacillus. Several workers have reported that catechins are inhibitory for $S$. mutans and Streptococcus sobrinus, with minimum inhibitory concentration ranging between 50 and $1000 \mu \mathrm{g} / \mathrm{ml}$, well within the concentrations found in brewed tea [26,30].

Suyama et al. [36] showed that consumption of green tea gum increases the resistance of enamel to acid, and reinforces its remineralization. This will culminate in the prevention of dental caries. Soekanto et al. [37], in his study, evaluated the students' saliva $\mathrm{pH}$ that had daily consumed green tea for a period of 1 month. He concluded that daily consumption of the green tea can significantly increase the saliva $\mathrm{pH}$ beyond the limit of $\mathrm{pH}=5.5$. Yoshiharu et al. [38] evaluated the influence of a bottled green tea beverage on dentin demineralization with a demineralization gel system. The green tea treatment showed a significantly lower mineral loss. The sugar-free bottled green tea beverage inhibited dentin lesion progression, presumably due to the effect of sub-ppm fluoride levels.

In several animal experiments and human trials, green tea and black tea have been shown to significantly reduce plaque scores and carries index [39-43]. Therefore, incorporation of green tea extract to dentifrices and mouth rinses, chewing gum will exhibit an antimicrobial and anticariogenic effect as it reduces the acidity of the saliva and plaque and it could be cost-effective caries prevention in developing countries. Despite abundant literature on the general health benefits of green tea exists, its effect on cariogenic microbes is limited. With this background, the aim of the present systematic review was to evaluate the effectiveness of green tea extract containing formulations on dental caries.

\section{METHODS}

\section{Structured question}

Does green tea extract containing formulations as a monotherapy or as an adjunct to mechanical oral hygiene has an effect on dental caries.

\section{Search strategy}

The databases of PubMed, Cochrane, Science direct, and Metapress were searched up to July 2015 for the related topic. We used free-text terms to search the following ongoing trial registers which include US National Institutes of Health Trials Register (http://clinicaltrials.gov), WHO Clinical Trials Registry Platform (http://apps.who.int/trialsearch/ default.aspx), and Clinical Trial Registry of India(http://ctri.nic. in/Clinicaltrials/advsearch.php). The search strategies included a combination of controlled vocabulary and free text terms (refer to the search terms in Table 1). We employed the keywords related to the intervention and outcome. Reference list of the identified randomized trials was also checked for possible additional studies

\section{Inclusion and exclusion criteria}

Randomized controlled trials and clinical trials evaluate the effectiveness of green tea extract containing formulations on cariogenic microflora and caries increment. Literature in other languages which can be translated by the reviewer was included. Studies are evaluating the anti-plaque effectiveness of green tea on gingivitis, periodontitis, oral malodor, and pericoronitis patients. The animal study, in vitro studies, in situ studies, Reviews and Literature in other languages which cannot be translated by the reviewer were excluded.

\section{Data collection and analysis}

Screening and selection: Electronic search was carried out using the keywords in the Search engines - PubMed (refer Appendix 1 for PubMed search strategy), Science Direct, Cochrane, Metapress, and Google Scholar which yielded a total of 437 articles which was entered in the flowchart (Fig. 1). Based on preset inclusion and exclusion criteria, the titles of the studies identified from the search were assessed independently by two review authors. Conflicts concerning the inclusion of the studies were resolved by discussion. 26 titles were identified from the search after excluding duplications. Abstracts of selected articles were reviewed independently. 19 articles were excluded after reading the abstract. Full-text articles were retrieved for seven relevant studies. After reviewing the articles independently, one article was excluded after full-text reading. Finally, six articles were selected based on eligibility criteria. The reference list of the fulltext articles was reviewed for identifying additional studies. Titles of articles relevant to the review were selected by discussion. Abstracts of the two selected articles were reviewed. The difference of opinion concerning the inclusion of a study was resolved by discussion and all two articles eliminated after reviewing abstracts. Quality Assessment criteria to evaluate the studies were decided by two review authors in accordance with CONSORT guidelines. The risk of bias for each study was independently assessed by the review authors and conflicts concerning the risk of bias were sorted by discussion.

\section{Data extraction}

Data extraction for general characteristics of studies and variables of outcome was done (Table 2). For each trial the following data were recorded which includes author, participants, and group, analyzed variables and evaluation methods, follow-up, and results. Each study's methodological quality was assessed (Fig. 2) using the domain-based evaluation described in the Higgins and green (Cochrane reviewer's handbook 2009). The study was assessed to have a "high risk" of bias if it did not record a "Yes" in three or more of the four main categories, "moderate risk" if two out of four categories did not record a "yes"

\section{Table 1: Electronic database search terms}

PubMed search terms: Dental caries OR caries dental OR dental decay OR decay dental OR carious dentin OR dentin, carious AND green tea OR green tea extract OR Camellia sinensis OR Sinensis camellia OR Sinenses camellia OR green tea catechins OR tea polyphenol OR green tea polyphenol OR green tea mouth rinse OR green tea mouthwash OR green tea chewing gum AND $S$. mutans OR anticariogenic effectiveness OR cariogenic microflora OR caries inhibitory effect.

Cochrane search terms: Green tea OR green tea extract OR Camellia sinensis OR Sinensis camellia OR green tea catechins OR tea polyphenol OR green tea polyphenol OR green tea mouthrinse OR green tea mouthwash OR green tea chewing gum AND $S$. mutans OR anticariogenic effectiveness OR cariogenic microflora OR caries inhibitory effect

Science direct search terms: Green tea mouth rinse OR green tea mouthwash OR green tea AND S. mutans OR dental caries

Metapress search terms: Dental caries AND green tea OR green tea extract AND S. mutans 


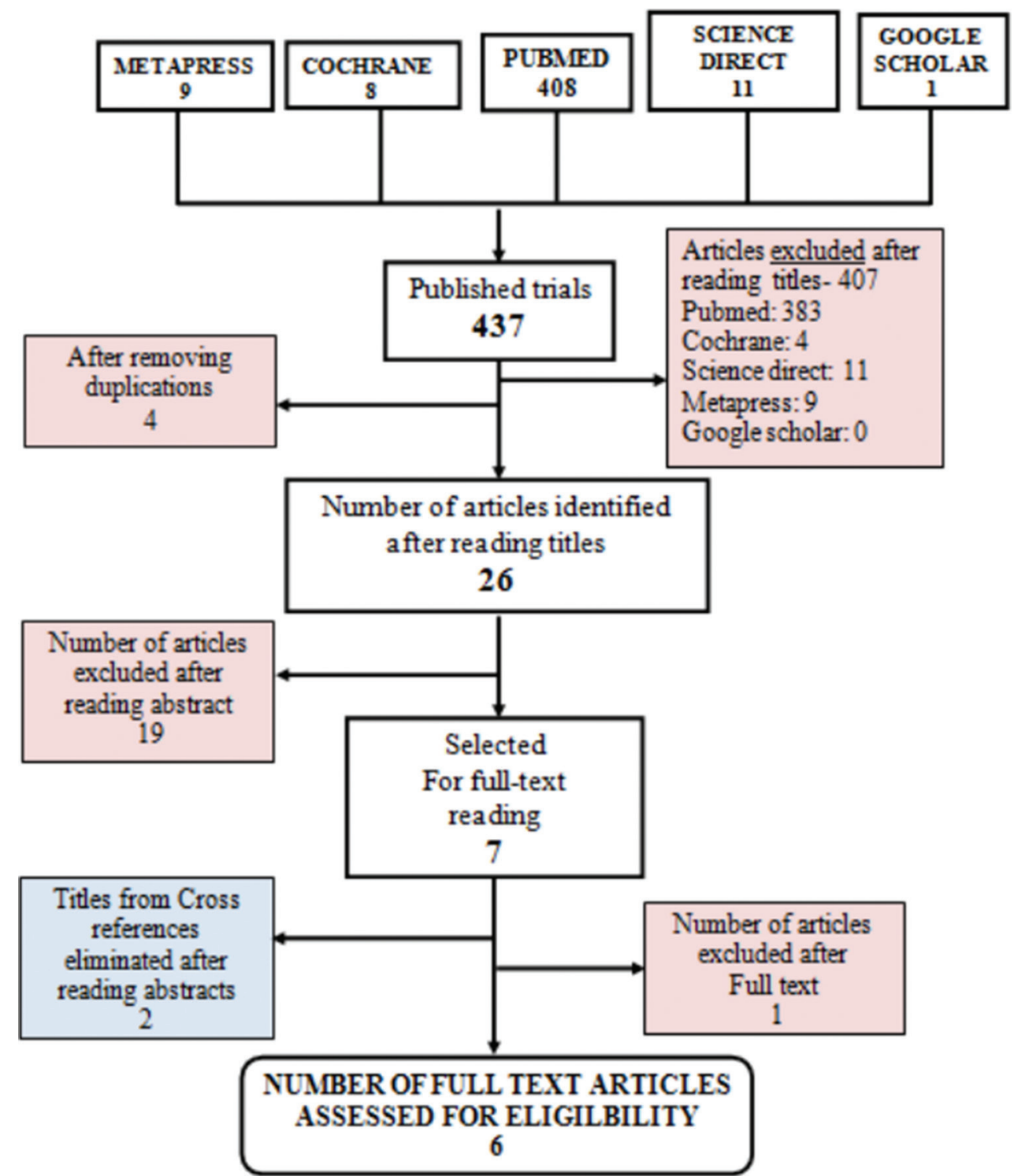

Fig. 1: Search strategy

and "low risk" if all the four categories recorded that is randomization assessor, blinding, and completeness of follow-up were considered adequate. In the case of nonrandomized and clinical trials without a control group, it is recorded as not applicable.

\section{Statistical analysis}

Due to the heterogeneity of the interventions and assessment of outcome, pooling of data was not possible.

\section{RESULTS}

The review included six studies, which assessed the effectiveness of green tea extract containing formulations on cariogenic microflora and caries increment $[32,34,35,44-46]$. Out of six studies included in this review, two studies provided data on the salivary concentration of S. mutans and Lactobacilli count $[34,35]$. One study provided data on Streptococcus mutans in both saliva and plaque [32]. One study focused on S. mutans in plaque alone [44]. One study focused on $S$. mutans in saliva [46], one study recorded the caries increment using DMFT and DMFS score [45].

Out of six studies, five studies [34,35,44-46] compared green tea with various vehicles as a control group (chlorhexidine, sodium fluoride, and placebo). One study used green tea as a test group without comparison [32].

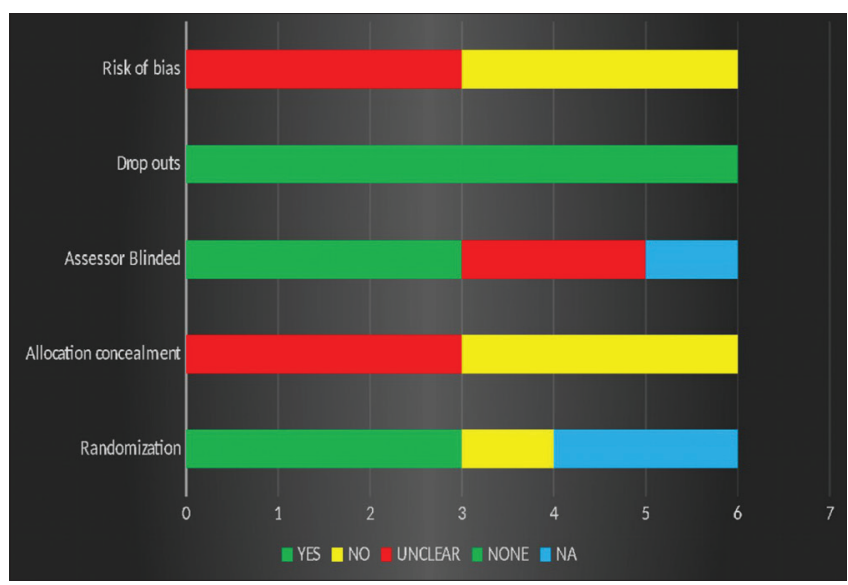

Fig. 2: Quality assessment of selected articles

One study compared green tea mouthrinse [44] with chlorhexidine and plain water. One study [34] compared green tea mouthrinse with placebo. One study [35] compared green tea mouthrinse with sodium fluoride. One study [45] compared green tea chewing gum with a 


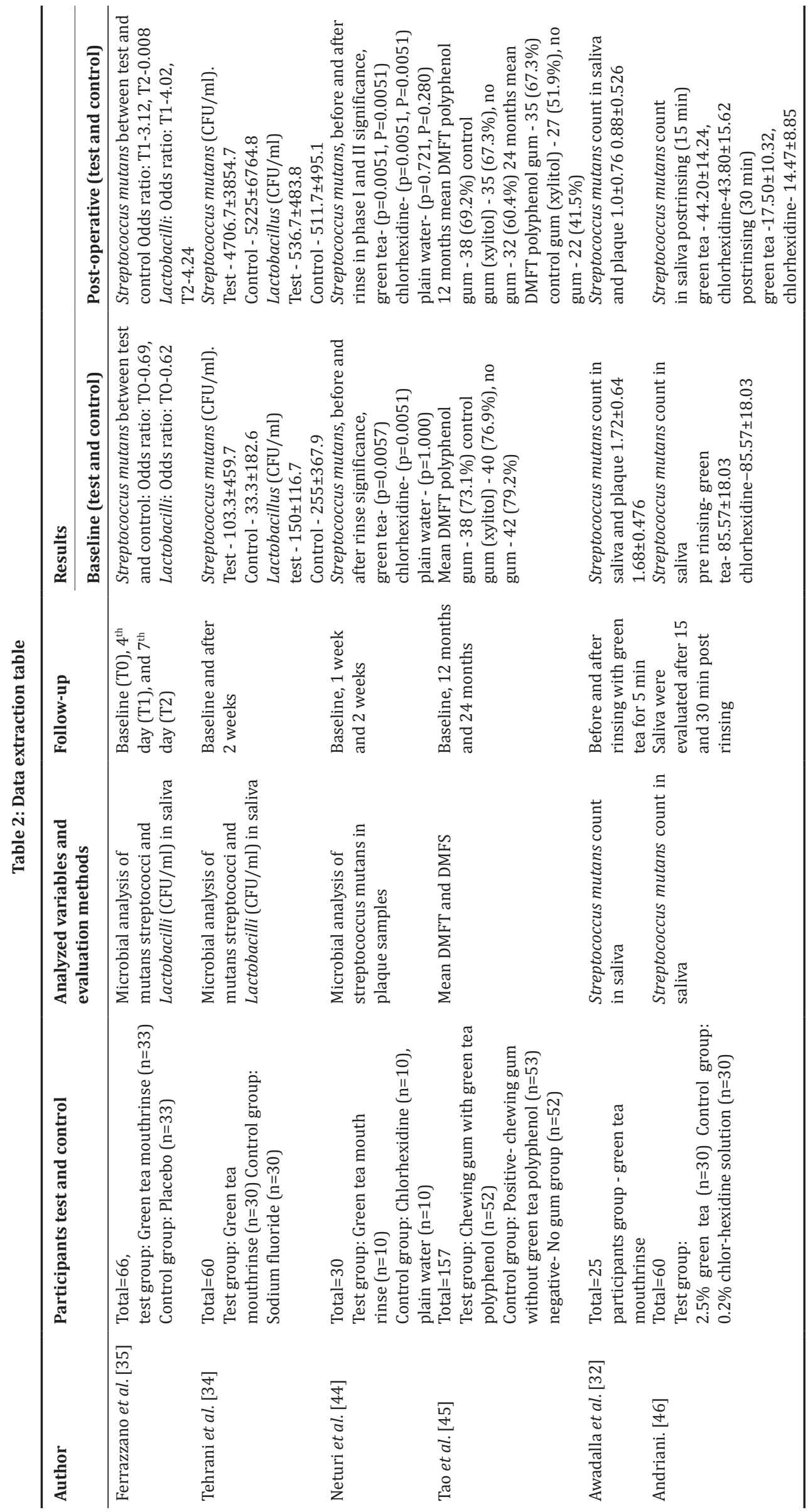


control group and no gum group. One study [46] compared green tea solution with chlorhexidine.

Four studies [32,34,35,44,46] used microbiological analysis for determination of cariogenic microorganisms in plaque and saliva. One study used the DMFT and DMFS index for assessing caries increment [45].

Out of the six studies, three studies $[35,44,45]$ had a low risk of bias and three studies had a high risk of bias $[34,35,46]$. High risk of bias in three studies due to unclear information on the randomization sequences generation or allocation or blinding which are important to overrule the selection bias, performance bias, and detection bias, respectively. Level of the evidence of selected articles was assessed based on the United States department of health and human services in 2012. Out of the six studies, three were randomized clinical trials and have level 1 evidence, one study was a quasi-experimental study, whereas the other two studies were clinical trials and have level 2 evidence.

\section{DISCUSSION}

Carbohydrates play an important role in the development of dental caries, especially when it remains on the tooth structure for a significant period of time $[47,48]$. Several studies have reported that fermentation of carbohydrates by bacteria mainly $S$. mutans results in a decrease in the $\mathrm{pH}$ of plaque and demineralization of enamel and finally formation of dental caries [49-52].

Several anticariogenic products have been found to be effective against the bacterial activity, out of which fluoride and chlorhexidine considered being the gold standard and established antimicrobial agents. However, the side effects of fluoride will be fluoride toxicity which occurs due to the risk of ingestion, and hence it is not recommended in small children, while chlorhexidine side effects are primarily staining and taste alteration, limit its potential to be used for the long-term purpose.

Due to these drawbacks, an increasing number of people all around the world are turning to nature using natural herbal products. There has been a change in thinking globally with a growing tendency to "go natural." The natural phytochemicals present in plants could offer an effective alternative to synthetic preparations. Research has been focused in recent years on herbal medicines due to their wide range of biological and medicinal activities, higher safety margins, and lower costs [32]. Green tea is a natural medicine which possesses a vital antibacterial effect and kills pathogens [53]. Furthermore, it has antioxidant, anti-inflammatory action [54]. Hence, the purpose of this systematic review is to evaluate the effectiveness of green tea extract containing formulations on dental caries.

The results of the studies included for review showed that green tea extract containing formulations when tested against a control showed positive results and induced a significant reduction in cariogenic microflora and DMFT score.

Considering the importance of cariogenic microflora in caries progression. Gianmaria et al. and Tehrani et al. reported green tea mouthrinse showed a significant reduction in $S$. mutans and Lactobacilli counts on microbial analysis of saliva. A pilot study done by Awadalla et al. showed a significant difference among subjects pre- and post-rinsing with $2 \%$ green tea for 5 min concerning $S$. mutans count in saliva and plaque. Neturi et al. reported chlorhexidine group showed a slightly greater significant reduction of $S$. mutans count in plaque than green tea mouth rinse. Fajriani et al., 2014, reported no statistically significant difference between the two groups $(\mathrm{p}=0.001)$; however, the decline in the chlorhexidine group was slightly greater than green tea.

\section{CONCLUSION}

With the available evidence, based on quality assessment and evidence level of selected articles, three studies had a high risk of bias with level 2 evidence and three studies had a low risk of bias with level 1evidence.
Therefore, it can be concluded that green tea extract containing formulations are effective in reducing cariogenic microflora and caries increment.

\section{Inference}

- Implications for practice: Green tea with its anticariogenic property and as it decreases the acidity of saliva and plaque, it can be used as a cost-effective caries preventive measure.

- Implications for research: Further studies must be performed with standard research methodology protocols with strict eligibility criteria, a combination of specific and sensitive detection methods, and reliable statistical analyses need to be addressed.

\section{AUTHORS' CONTRIBUTION}

Dr. P. Jayashri has provided a substantial contribution to the concepts, design, the definition of intellectual content, literature search, Data acquisition, Data analysis, Statistical analysis, Manuscript preparation, editing and review, and the guarantor. Dr. Joseph John has made a substantial contribution to study conception and design, Acquisition of data, Analysis and Interpretation of data, Manuscript drafting, and critical revision.

Dr. Meignana Arumugham I, has a major role in study conception and design, Acquisition of data, Analysis and Interpretation of data, Manuscript drafting and critical revision. Dr. R. Pradeep Kumar has contributed to the Statistical analysis, Manuscript preparation, editing, and review. Dr. D. Srisakthi has contributed to the Statistical analysis, Manuscript preparation, editing, and review.

\section{CONFLICTS OF INTEREST}

The authors declare that there are no conflicts of interest regarding publication of this article.

\section{REFERENCES}

1. Campus G, Condò SG, Di Renzo G, Ferro R, Gatto R, Giuca MR, et al. National Italian guidelines for caries prevention in 0 to 12 years-old children. Eur J Paediatr Dent 2007;8:153-9.

2. Damle SC, Patel AR. Caries prevalence and treatment need amongst children of Dharavi, Bombay, India. Community Dent Oral Epidemiol 1994:22:62-3

3. Tewari A, Chawla HS. Study of prevalence of dental caries in an urban area of India (Chandigarh). J Indian Dent Assoc 1977:49:231-9.

4. Dash JK, Sahoo PK, Bhuyan SK, Sahoo SK. Prevalence of dental caries and treatment needs among children of Cuttack (Orissa). J Indian Soc Pedod Prev Dent 2002;20:139-43.

5. Dhar V, Jain A, Van Dyke TE, Kohli A. Prevalence of dental caries and treatment needs in the school-going children of rural areas in Udaipur district. J Indian Soc Pedod Prev Dent 2007;25:119-21.

6. Saravanan S, Kalyani V, Vijayarani MP, Jayakodi P, Felix J, Arunmozhi $\mathrm{P}$, et al. Caries prevalence and treatment needs of rural school children in Chidambaram Taluk, Tamil Nadu, South India. Indian J Dent Res 2008;19:186-90.

7. Keyes PH. Present and future measures for dental caries control. J Am Dent Assoc 1969;79:1395-404.

8. Hamada S, Slade HD. Biology, immunology, and cariogenicity of Streptococcus mutans. Microbiol Rev 1980;44:331-84.

9. Nayak SS, Kumar BR, Ankola AV, Hebbal M. The efficacy of Terminalia chebula rinse on Streptococcus mutans count in saliva and its effect on salivary pH. Oral Health Prev Dent 2010;8:55-8.

10. Chatterjee A, Saluja M, Agarwal G, Alam M. Green tea: A boon for periodontal and general health. J Indian Soc Periodontol 2012;16:161-7.

11. Subramaniam P, Eswara U, Maheshwar Reddy KR. Effect of different types of tea on Streptococcus mutans: An in vitro study. Indian J Dent Res 2012;23:43-8.

12. Featherstone JD. The science and practice of caries prevention. J Am Dent Assoc 2000;131:887-99.

13. Heymann HO, Ritter AV. Sturdevant's Art and Science of Operative Dentistry. St. Louis: Elsevier Health Sciences; 2014.

14. Herrera D, Santos S, Ferrús J, Barbieri G, Trombelli L, Sanz M, et al. Efficacy of a $0.15 \%$ benzydamine hydrochloride and $0.05 \%$ cetylpyridinium chloride mouth rinse on 4-day de novo plaque 
formation. J Clin Periodontol 2005;32:595-603.

15. Namita P, Mukesh R, Vijay K. Camellia sinensis (Green tea): A review. Glob J Pharm 2012;6:529.

16. Taylor PW, Hamilton-Miller JM, Stapleton PD. Antimicrobial properties of green tea catechins. Food Sci Technol Bull 2005;2:71-81.

17. Lobo PL, de Carvalho CB, Fonseca SG, de Castro RS, Monteiro AJ, Fonteles MC, et al. Sodium fluoride and chlorhexidine effect in the inhibition of Mutans streptococci in children with dental caries: A randomized, double-blind clinical trial. Oral Microbiol Immunol 2008;23:486-91.

18. Burt BA. Prevention policies in the light of the changed distribution of dental caries. Acta Odontol Scand 1998;56:179-86.

19. Murray JJ, Rugg-Gunn AJ, Jenkins GN, editors. A history of water fluoridation. In: Fluorides in Caries Prevention. Oxford: Wright; 1991. p. 7-37.

20. Horowitz HS. The need for toothpastes with lower than conventional fluoride concentrations for preschool-aged children. J Public Health Dent 1992;52:216-21.

21. Stookey GK. Review of fluorosis risk of self-applied topical fluorides: Dentifrices, mouthrinses and gels. Community Dent Oral Epidemiol 1994;22:181-6.

22. Bentley EM, Ellwood RP, Davies RM. Fluoride ingestion from toothpaste by young children. Br Dent J 1999;186:460-2.

23. Rojas-Sanchez F, Kelly SA, Drake KM, Eckert GJ, Stookey GK, Dunipace AJ, et al. Fluoride intake from foods, beverages and dentifrice by young children in communities with negligibly and optimally fluoridated water: A pilot study. Community Dent Oral Epidemiol 1999;27:288-97.

24. Levy SM. Review of fluoride exposures and ingestion. Community Dent Oral Epidemiol 1994;22:173-80.

25. Lewis DW, Limeback H. Comparison of recommended and actual mean intakes of fluoride by Canadians. J Can Dent Assoc 1996;62:7089, 712-5.

26. Hrishi TS, Kundapur PP, Naha A, Thomas BS, Kamath S, Bhat GS, et al. Effect of adjunctive use of green tea dentifrice in periodontitis patients - A randomized controlled pilot study. Int J Dent Hyg 2016;14:178-83.

27. Miller JM. Anticariogenic properties of tea (Camellia sinensis). J Med Micobiol 2001;50:299-302

28. Rasheed A, Haider M. Antibacterial activity of Camellia sinensis extracts against dental caries. Arch Pharm Res 1998;21:348-52.

29. Smullen J, Koutsou GA, Foster HA, Zumbé A, Storey DM. The antibacterial activity of plant extracts containing polyphenols against Streptococcus mutans. Caries Res 2007;41:342-9.

30. Hassani AS, Amirmozafari N, Ordouzadeh N, Hamdi K, Nazari R, Ghaemi A, et al. Volatile components of Camellia sinensis inhibit growth and biofilm formation of oral streptococci in vitro. Pak J Biol Sci 2008;11:1336-41.

31. Hirasawa M, Takada $\mathrm{K}$, Otake S. Inhibition of acid production in dental plaque bacteria by green tea catechins. Caries Res 2006;40:265-70.

32. Awadalla HI, Ragab MH, Bassuoni MW, Fayed MT, Abbas MO. A pilot study of the role of green tea use on oral health. Int J Dent Hyg 2011;9:110-6

33. Venkateswara B, Sirisha K, Chava VK. Green tea extract for periodontal health. J Indian Soc Periodontol 2011;15:18-22.

34. Tehrani MH, Asghari G, Hajiahmadi M. Comparing Streptococcus mutans and Lactobacillus colony count changes following green tea mouth rinse or sodium fluoride mouth rinse use in children (Randomized double-blind controlled clinical trial). Dent Res J (Isfahan) 2011;8:S58-63.

35. Ferrazzano GF, Roberto L, Amato I, Cantile T, Sangianantoni G, Ingenito A, et al. Antimicrobial properties of green tea extract against cariogenic microflora: An in vivo study. J Med Food 2011;14:907-11.

36. Suyama E, Tamura T, Ozawa T, Suzuki A, Iijima Y, Saito T, et al. Remineralization and acid resistance of enamel lesions after chewing gum containing fluoride extracted from green tea. Aust Dent J 2011;56:394-400.

37. Soekanto SA, Mangundjaja S, Djais A. Effect of Green Tea on Canes Activity of Mutans Streptococci. Ho Chi Minh City: Presented at the $2^{\text {nd }}$ International Dental Collaboration of the Mekong River Region in Conjunction with The $27^{\text {th }}$ Annual Scientific Meeting of Faculty Odonto-Stomatology HCM City; 2005.

38. Yoshiharu M, Kazuko K, Yukio H, Toshio T. Anti-demineralizing potential of bottled sugar-free green tea beverages in vitro. Oral Sci Int 2009; 6:21-6.

39. Onisi M, Ozaki F, Yoshino F, Murakami Y. An experimental evidence of caries preventive activity of non-fluoride component in tea. Koku Eisei Gakkai Zasshi 1981;31:158-62.

40. Rosen S, Elvin-Lewis M, Beck FM, Beck EX. Anticariogenic effects of tea in rats. J Dent Res 1984;63:658-60.

41. Elvin-Lewis M, Steelman R. The anticariogenic effects of tea drinking among Dallas school children. J Dent Res 1986;65:198.

42. Ooshima T, Minami T, Aono W, Tamura Y, Hamada S. Reduction of dental plaque deposition in humans by oolong tea extract. Caries Res 1994;28:146-9.

43. Kempler D, Anaise J, Westreich V, Gedalia I. Caries rate in hamsters given non acidulated and acidulated tea. J Dent Res 1977;56:89-94.

44. Neturi RS, R S, B VS, Y SS, T CS, P SK, et al. Effects of green tea on Streptococcus mutans counts - A randomised control trail. J Clin Diagn Res 2014;8:ZC128-30.

45. Tao DY, Shu CB, Lo EC, Lu HX, Feng XP. A randomized trial on the inhibitory effect of chewing gum containing tea polyphenol on caries. J Clin Pediatr Dent 2013;38:67-70.

46. Jennifer F, Andriani N. Reduction of salivary Streptococcus mutans colonies in children after rinsing with $2.5 \%$ green tea solution. J Dent Indones 2014;21:81-6.

47. Anusavice KJ. Caries risk assessment. Oper Dent 2001;6:19-26.

48. Ekstrand KR. Occlusal caries: Pathology, diagnosis, and logical management. Dent Update 2001;28:380-7.

49. Mundorff SA, Bibby BG. Cariogenic potential of foods. Caries Res 1990;24:344-9.

50. Clarkson BH. Introduction to cariology: The discipline of cariology; art or science. Dent Clin N Am 1999;43:569-77.

51. Baneriee A, Watson TF, Kidd EA. Dentin caries: Take it or leave it? Dent Update 2000;27:272-6.

52. Ramya R, Srinivasan R. Clinical Operative Dentistry, Principles and Practice. $1^{\text {st }}$ ed. Bangalore, India. EMMESS Medical Publishers; 2007. p. $53-79$

53. Rahmani A, Aldebasi YH, Aly SM. Role of green tea and its constituent epigallocatechin-3-gallate in the health management. Int $\mathrm{J}$ Pharm Pharm Sci 2015;7:6-12.

54. Rathod SJ, Williamson M. Antibacterial activity of green tea extract in combination with cefotaxime on diarrhea causing esbl producing E. coli. Int J Pharm Pharm Sci 2015;7:258-62. 
Appendix 1: PubMed search strategy

\begin{tabular}{lll}
\hline Number & Search history & Articles (n) \\
\hline 1 & "Dental caries" & 47930 \\
2 & "Caries dental" & 47930 \\
3 & "Dental decay" & 48581 \\
4 & "Decay dental" & 48581 \\
5 & "Carious dentin" & 48335 \\
6 & "Dentin, carious" & 48335 \\
7 & \#1 or \#2 or \#3 or \#4 or \#5 or\#6 & 793 \\
8 & "Green tea" & 6410 \\
9 & "Green tea extract" & 1385 \\
10 & "Camellia sinensis" & 2101 \\
11 & "Sinensis camellia" & 2101 \\
12 & "Sinensis camellia" & 0 \\
13 & "Green tea catechins" & 2961 \\
14 & "Tea polyphenol" & 2051 \\
15 & "Green tea polyphenol" & 7511 \\
16 & "Green tea mouth rinse" & 5 \\
17 & "Green tea mouthwash" & 29 \\
18 & "Green tea chewing gum" & 7 \\
19 & \#8 or \#9 or \#10 or \#11 or \#12 or\#13 & 8334 \\
& or\#14 or\#15 or\#16 or\#17 or\#18 & \\
20 & "Streptococcus mutans" & 9457 \\
21 & "Anticariogenic effectiveness" & 16 \\
22 & "Cariogenic microflora" & 87 \\
23 & "Caries inhibitory effect" & 271 \\
24 & \#20 or\#21 or\#22 or\#23 & 9600 \\
25 & \#7 and \#19 and \#24 & 408 \\
\hline
\end{tabular}

